\title{
Safety and Efficacy of Zoledronic Acid Treatment with and without Acetaminophen and Eldecalcitol for Osteoporosis
}

\author{
Takeshi Mochizuki ${ }^{1}$, Koichiro Yano ${ }^{2}$, Katsunori Ikari ${ }^{2}$ and Ken Okazaki ${ }^{2}$
}

\begin{abstract}
:
Objectives We aimed to investigate the safety of zoledronic acid (ZOL) combined with acetaminophen (APAP) regarding both the adverse events and the efficacy of ZOL combined with an eldecalcitol (ELD) in a randomized clinical trial conducted in patients with primary osteoporosis.

Methods A total of 109 patients were administered ZOL $5 \mathrm{mg}$ and then were randomly assigned to the following groups (3:2:1): those treated with ZOL, those treated with ZOL combined with APAP and ELD, and those treated with ZOL combined with ELD. For the analyses, the groups were classified into four treatment groups: patients treated with APAP (APAP group) and without APAP (non-APAP group), and those treated with ELD (ELD group) and without ELD (non-ELD group). The incidence rates of symptomatic adverse events were compared between the APAP and non-APAP groups, and the efficacy was compared between the ELD and non-ELD groups.

Results In the APAP and non-APAP groups, the incidence rates of symptomatic adverse events were $20.6 \%$ and $44.6 \%$ ( $\mathrm{p}=0.009$ ), respectively. Age and APAP use were found to be significant factors associated with adverse events. The percent changes in the bone mineral density values from baseline ( $\triangle \mathrm{BMD}$ ) in the ELD and non-ELD groups at 12 months were $8.2 \%$ and $6.2 \%$ for the lumbar spine, $4.2 \%$ and $4.0 \%$ for the total hip, and $3.9 \%$ and $2.2 \%$ for the femoral neck, respectively. The $\triangle \mathrm{BMD}$ of all sites did not differ significantly between the ELD and non-ELD groups.

Conclusion In ZOL treatment, the co-administration of APAP should thus be considered as a therapeutic option to reduce the occurrence of symptomatic adverse events stemming from ZOL treatment in Japanese patients with primary osteoporosis, particularly in younger patients.
\end{abstract}

Key words: acetaminophen, adverse event, eldecalcitol, osteoporosis, zoledronic acid

(Intern Med 60: 2585-2591, 2021)

(DOI: 10.2169/internalmedicine.6607-20)

\section{Introduction}

Osteoporosis is a systemic bone disease characterized by a low bone mass and bone quality, which can lead to fragility fractures. The most common fragility fractures are vertebral and hip fractures. Vertebral and hip fractures are associated with increased mortality (1-6). In Japan, there was a drastic increase in the number of patients with hip fractures from 2009 to 2014 (7). This is probably due to fact that the Japanese population is rapidly aging. Although oral bisphosphonate is the gold standard in the treatment of osteoporo- sis, poor adherence to oral bisphosphonate treatment has become a growing problem due to the administration methods. Poor adherence to osteoporosis treatment increases the risk of fragility fractures $(8,9)$.

Zoledronic acid (ZOL) is a bisphosphonate treatment administered by infusion at a dose of $5 \mathrm{mg}$ once yearly. ZOL can reduced bone turnover, increase the bone mineral density (BMD), and reduce the risk of vertebral and/or hip fractures (10-14). However, major adverse events associated with ZOL treatment are observed in Japanese patients, including pyrexia, nasopharyngitis, and arthralgia (13). Previous studies have shown that administration of ZOL with

${ }^{1}$ Department of Rheumatology and Orthopaedic Surgery, Kamagaya General Hospital, Japan and ${ }^{2}$ Department of Orthopaedic Surgery, Tokyo Women's Medical University, Japan

Received for publication October 28, 2020; Accepted for publication January 18, 2021

Correspondence to Dr. Takeshi Mochizuki, twmutamo@gmail.com 
acetaminophen (APAP) reduced pyrexia in comparison to ZOL with a placebo $(15,16)$. Oral bisphosphonate combined with eldecalcitol (ELD) resulted in a higher BMD increase in comparison to oral bisphosphonate monotherapy (17).

In Japan, the efficacy and safety information of treatment with ZOL in Japanese patients are insufficient. Hence, the efficacy and safety of this treatment should be validated in Japanese patients with osteoporosis. Therefore, we aimed to investigate the safety of ZOL combined with APAP in terms of adverse events, and the efficacy of ZOL combined with ELD in a randomized clinical trial.

\section{Materials and Methods}

\section{Patients and treatment}

The present study was a randomized open-label clinical trial. A total of 109 patients who were outpatients at our hospital, were enrolled in this study between June 2017 and June 2019. The patients had a young adult mean (YAM) of $\leq 70 \%$ at the lumbar spine and/or total hip based on dual energy X-ray absorptiometry (DXA) measurements, or a previous fragility fracture of either the lumbar spine or the proximal femur and a YAM of $<80 \%$. The exclusion criteria were as follows: a history of treatment for osteoporosis and glucocorticoid-induced osteoporosis. The patients were administered $5 \mathrm{mg}$ of ZOL by intravenous infusion and then were randomly assigned by the clinical trial center of our institution in a 3:2:1 ratio into the following groups: those treated with monotherapy (ZOL mono group), those treated with ZOL combined with APAP and ELD (ZOL+APAP+ ELD group), and those treated with ZOL combined with ELD (ZOL+ELD group). An APAP $500 \mathrm{mg}$ tablet was administered before ZOL infusion and thrice daily for 3 days. ELD, an active vitamin D3 analog, was used daily at doses of $0.5-0.75 \mu \mathrm{g}$. Patients with kidney dysfunction were administrated eldecalcitol of $0.5 \mu \mathrm{g}$. ZOL treatment was initiated after confirming the absence of pyrexia.

\section{Safety assessment}

Patients' temperature was measured three times a day during the first 3 days after ZOL administration. Pyrexia was defined as a temperature above $37.5^{\circ} \mathrm{C}(15)$. The axillary temperature was measured three times a day for 3 days and recorded in a self-report form. The maximum temperature was also recorded. The albumin-adjusted serum calcium (Ca) levels were measured at baseline and at 3, 6, and 12 months. The patients received daily oral supplements of $\mathrm{Ca}$ at a dose of $600 \mathrm{mg}$ if the serum Ca level was $<8.8 \mathrm{mg} / \mathrm{dL}$. ELD administration was discontinued when the serum $\mathrm{Ca}$ level was $>10.4 \mathrm{mg} / \mathrm{dL}$.

\section{Efficacy assessment}

The BMD values of the lumbar spine, total hip, and femoral neck were measured by DXA using the Prodigy
System (GE Healthcare, Madison, USA) at baseline and at 3, 6, and 12 months. Vertebral fractures were evaluated by plain X-rays from the T8 vertebra to the lumbar spine at baseline and 12 months. A new vertebral fracture was defined as an increase of at least 1 on a semiquantitative grading scale and a worsening fracture as a height loss of $\geq 20 \%$ at the vertebra (13). The bone turnover makers of procollagen type I N-terminal propeptide (PINP) and tartrateresistant acid phosphatase-5b (TRACP-5b) were recorded at baseline and at 3, 6, and 12 months.

\section{Statistical analysis}

A safety and efficacy analysis was performed for patients who visited at least once after ZOL infusion (Fig. 1). The demographic characteristics of the ZOL mono, ZOL+APAP+ ELD, and ZOL+ELD groups were compared using an analysis of variance. Moreover, the demographic characteristics of ZOL combined with APAP (APAP group) and ZOL without APAP (non-APAP group) and those of ZOL with ELD (ELD group) and ZOL without ELD (non-ELD group) were compared using the Mann-Whitney $U$ and Fisher's exact tests. The rate of symptomatic adverse events was compared between the APAP and non-APAP groups. The factors associated with adverse events were analyzed by a univariate analysis. Moreover, a multivariate logistic regression analysis was performed using the variables with a $\mathrm{p}$ value of $<0.1$ identified by a univariate analysis. The cutoff values of related factors in safety were measured using the receiver operating characteristic (ROC) method with corresponding sensitivity and specificity as well as the area under the curve (AUC). The rate of an abnormal Ca level was compared between the ELD and non-ELD groups. The percent changes $(\Delta)$ in BMD at 3, 6, and 12 months from baseline and bone turnover makers were analyzed using the paired $t$-test. The efficacy of ZOL was compared between the ELD and nonELD groups. A $p$ value of $<0.05$ was considered to be significant.

\section{Results}

The baseline demographics and clinical characteristics in the ZOL mono, ZOL+APAP+ELD, and ZOL+ELD groups are summarized in Table 1a. There was no difference in all variables. The baseline demographics and clinical characteristics of the APAP and non-APAP groups are summarized in Table 1b. The PINP value in the APAP group was significantly lower than that of the non-APAP group. The baseline demographics and clinical characteristics of the ELD and non-ELD groups are summarized in Table 1c. There was no difference in any of the variables. The rate of ELD dose of $0.5 \mu \mathrm{g}$ was $28.1 \%$ because of renal dysfunction.

\section{Safety}

In the ZOL mono group, $46.9 \%$ patients exhibited symptomatic adverse events. In the APAP group, $20.6 \%$ of the patients had symptomatic adverse events. The symptomatic 


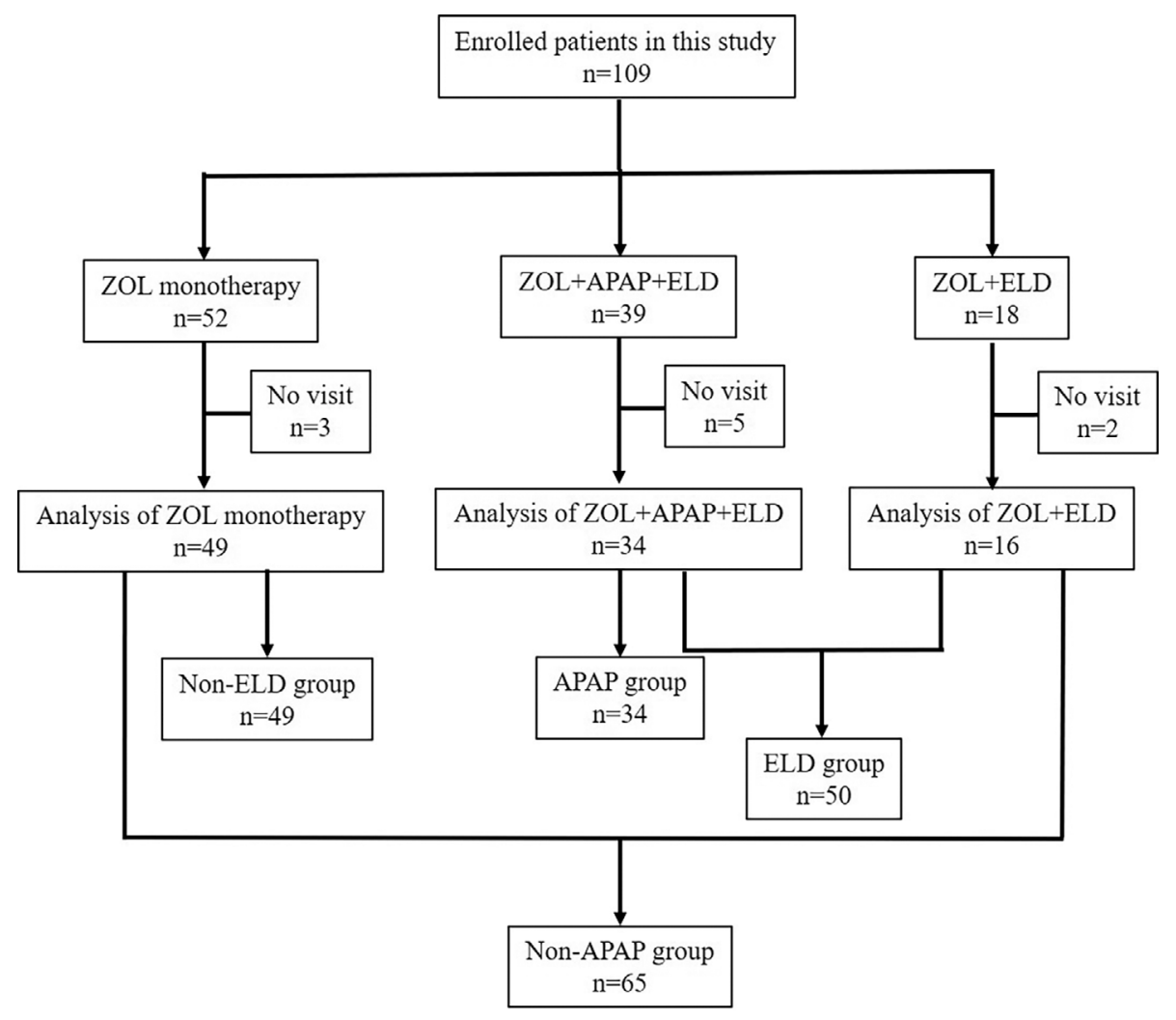

Figure 1. Patient enrollment and groups.

adverse events included pyrexia, arthralgia, headache, chest pain, and hyperemia in $5,1,1,1$, and 1 patients, respectively. In the non-APAP group, $44.6 \%$ of the patients had symptomatic adverse events. The symptomatic adverse events included pyrexia, arthralgia, fatigue, appetite loss, myalgia, headache, hypertension, nausea, diarrhea, and dizziness in $19,3,2,2,2,1,1,1,1$, and 1 patients, respectively. The number of patients and the day of onset in patients with pyrexia were 2 patients at 0 day, 17 patients at 1 day, and 5 patients at 2 day, respectively. The APAP group had a significantly lower rate of symptomatic adverse events than the non-APAP group ( $\mathrm{p}=0.009)$. Table 2 shows the results of comparisons between the patients with and without adverse events regarding demographic characteristics at baseline by using univariate analysis. The age and APAP use were significant factors, and a multivariate analysis confirmed age and APAP use to be significant factors (Table 3). The cutoff value of age calculated by the ROC method was 82.0 (sensitivity: $23.1 \%$, specificity: $77.1 \%$, AUC: 0.368 ).

Hypocalcemia and hypercalcemia were observed each in 3 patients (5.3\% each) in the ELD group and each in 1 patient $(1.9 \%$ each) in the non-ELD group. One patient in each of the two groups was prescribed oral calcium 1aspartate hydrate $600 \mathrm{mg}$, and two patients in the ELD group were discontinued on ELD. The rate of abnormal serum $\mathrm{Ca}$ levels did not differ significantly between these two groups $(\mathrm{p}=0.618)$.

\section{Efficacy \\ Changes in BMD}

$\triangle \mathrm{BMD}$ values at 3,6 , and 12 months in the ELD group significantly increased by $4.6 \% \pm 5.0 \% \quad(\mathrm{p}<0.001), 6.6 \% \pm$ $5.0 \%(\mathrm{p}<0.001)$, and $8.2 \% \pm 5.1 \%(\mathrm{p}<0.001)$ for the lumbar spine; $1.8 \% \pm 3.3 \% \quad(\mathrm{p}=0.016), 2.4 \% \pm 7.4 \% \quad(\mathrm{p}=0.002)$, and $4.2 \% \pm 6.0 \%(\mathrm{p}<0.001)$ for the total hip; and $1.5 \% \pm 5.5 \%$, $3.0 \% \pm 4.9 \%$, and $3.9 \% \pm 9.1 \%(\mathrm{p}=0.018)$ for the femoral neck from baseline, respectively (Fig. 2). The $\triangle \mathrm{BMD}$ values at 3 , 6 , and 12 months in the non-ELD group significantly increased by $3.5 \% \pm 3.2 \% \quad(\mathrm{p}<0.001), 5.4 \% \pm 4.5 \% \quad(\mathrm{p}<0.001)$, and $6.2 \% \pm 5.6 \%(\mathrm{p}<0.001)$ for the lumbar spine; $2.6 \% \pm 3.9 \%$ $(\mathrm{p}=0.001), 2.6 \% \pm 3.9 \% \quad(\mathrm{p}=0.002)$, and $4.0 \% \pm 3.6 \% \quad(\mathrm{p}<$ 0.001 ) for the total hip; and $1.7 \% \pm 7.3 \%, 1.7 \% \pm 6.9 \%$, and $2.2 \% \pm 6.6 \%$ for the femoral neck from baseline, respectively (Fig. 2). The $\triangle \mathrm{BMD}$ for the lumbar spine, total hip, and femoral neck did not differ significantly between these two groups at any time point.

\section{Changes in bone turnover markers levels}

The change values at 3,6, and 12 months relative to the baseline in the ELD group were $-61.0 \% \pm 21.2 \%,-64.6 \% \pm$ $22.8 \%$, and $-62.1 \% \pm 24.5 \%$ for P1NP; and $-63.3 \% \pm 12.5 \%$, $-61.7 \% \pm 12.4 \%$, and $-60.1 \% \pm 13.6 \%$ for TRACP-5b, respectively. The change values at 3,6 , and 12 months relative to the baseline in the non-ELD group were $-59.2 \% \pm 29.1 \%$, $60.4 \% \pm 24.1 \%$, and $-47.6 \% \pm 36.1 \%$ for P1NP; and $-53.8 \% \pm$ $19.6 \%,-50.1 \% \pm 19.4 \%$, and $-45.6 \% \pm 22.2 \%$ for TRACP-5b, respectively. In both groups, $\triangle \mathrm{P} 1 \mathrm{NP}$ and $\triangle \mathrm{TRACP}-5 \mathrm{~b}$ sig- 
Table 1. Demographic Characteristics at Baseline of the (a) Zoledronic Acid Mono, Zoledronic Acid+acetami nophen+eldecalcitol, and Zoledronic Acid+eldecalcitol Groups, (b) Acetaminophen and Non-acetaminophen Groups, and (c) Eldecalcitol and Non-eldecalcitol Groups; Univariate Analysis.

\begin{tabular}{lcccc}
\hline (a) & & & \\
\hline \multicolumn{1}{c}{ Variables, median (Q1, Q3) } & $\begin{array}{c}\text { ZOL mono group } \\
(\mathrm{n}=52)\end{array}$ & $\begin{array}{c}\text { ZOL+APAP+ELD group } \\
(\mathrm{n}=39)\end{array}$ & $\begin{array}{c}\text { ZOL+ELD group } \\
(\mathrm{n}=18)\end{array}$ & $\mathrm{p}$ value \\
\hline Age, years & $75(70,83)$ & $75(72,81)$ & $78(72,83)$ & 0.777 \\
Female, $\mathrm{n}(\%)$ & $48(92.3)$ & $37(94.9)$ & $17(94.4)$ & 0.878 \\
Body weight, kg & $49.8(45,55)$ & $52(44.8,55)$ & $49(44.5,59.5)$ & 0.644 \\
Cr-eGFR, mL/min/1.73m ${ }^{2}$ & $68.55(60.2,78.7)$ & $66.7(58.5,76.7)$ & $68.8(60.63,78.8)$ & 0.499 \\
Value of serum calcium, mg/dL & $9.6(9.28,9.73)$ & $9.6(9.4,9.8)$ & $9.4(9.2,9.7)$ & 0.220 \\
Presence of vertebral fractures, $\mathrm{n}(\%)$ & $26(50)$ & $19(48.7)$ & $9(50)$ & 1.000 \\
Lumbar spine BMD, g/cm ${ }^{2}$ & $0.838(0.756,0.905)$ & $0.795(0.715,0.869)$ & $0.766(0.709,0.885)$ & 0.265 \\
Lumbar spine T score & $-2.35(-2.9,-1.75)$ & $-2.6(-3.2,-2.05)$ & $-2.7(-3.18,-1.9)$ & 0.254 \\
Total hip BMD, g/cm ${ }^{2}$ & $0.641(0.587,0.679)$ & $0.666(0.585,0.718)$ & $0.664(0.595,0.678)$ & 0.729 \\
Total hip T score, mean (SD) & $-2.5(-2.9,-2.18)$ & $-2.3(-2.95,-1.85)$ & $-2.3(-2.8,-2.2)$ & 0.617 \\
Femoral neck, g/cm ${ }^{2}$ & $0.617(0.576,0.682)$ & $0.615(0.559,0.664)$ & $0.621(0.564,0.681)$ & 0.632 \\
Femoral neck T score & $-2.8(-3.2,-2.28)$ & $-2.8(-3.4,-2.45)$ & $-2.65(-3.2,-2.05)$ & 0.388 \\
P1NP, ng/mL & $68.85(46.2,84.4)$ & $63.3(44.85,75.3)$ & $82.8(68.08,100.1)$ & 0.210 \\
TRACP-5b, mU/dL & $511.5(420.5,656.5)$ & $573(445,642.5)$ & $627(449.75,827)$ & 0.095 \\
\hline
\end{tabular}

\begin{tabular}{lccc}
\hline (b) & & & \\
\hline \multicolumn{1}{c}{ Variables, median (Q1, Q3) } & APAP group $(\mathrm{n}=39)$ & Non-APAP group $(\mathrm{n}=70)$ & $\mathrm{p}$ value \\
\hline Age, years & $75(72,81)$ & $75(70.25,83)$ & 0.839 \\
Female, $\mathrm{n}(\%)$ & $37(94.9)$ & $65(92.9)$ & 1.000 \\
Body weight, kg & $52(44.8,55)$ & $49.8(45,55)$ & 0.353 \\
Cr-eGFR, mL/min/1.73m ${ }^{2}$ & $66.7(58.5,76.7)$ & $68.7(60.3,79.5)$ & 0.712 \\
Value of serum calcium, mg/dL & $9.6(9.4,9.8)$ & $9.6(9.4,9.8)$ & 0.116 \\
Presence of vertebral fractures, $\mathrm{n}(\%)$ & $19(48.7)$ & $35(50)$ & 1.000 \\
Lumbar spine BMD, g/cm ${ }^{2}$ & $0.795(0.715,0.869)$ & $0.821(0.746,0.904)$ & 0.319 \\
Lumbar spine T score & $-2.6(-3.2,-2.05)$ & $-2.5(-3,-1.8)$ & 0.277 \\
Total hip BMD, g/cm ${ }^{2}$ & $0.666(0.585,0.718)$ & $0.648(0.59,0.678)$ & 0.426 \\
Total hip T score, mean (SD) & $-2.3(-2.95,-1.85)$ & $-2.5(-2.9,-2.2)$ & 0.418 \\
Femoral neck, g/cm ${ }^{2}$ & $0.615(0.559,0.664)$ & $0.617(0.571,0.683)$ & 0.381 \\
Femoral neck T score & $-2.8(-3.4,-2.45)$ & $-2.75(-3.2,-2.2)$ & 0.237 \\
P1NP, ng/mL & $63.3(44.85,75.3)$ & $72.45(54.45,92.15)$ & 0.043 \\
TRACP-5b, mU/dL & $573(445,642.5)$ & $517.5(422.25,708)$ & 0.626 \\
\hline
\end{tabular}

(c)

\begin{tabular}{lccc}
\hline \multicolumn{1}{c}{ Variables, median (Q1, Q3) } & ELD group $(\mathrm{n}=57)$ & Non-ELD group $(\mathrm{n}=52)$ & p value \\
\hline Age, years & $75(72,81)$ & $75(70,83)$ & 0.549 \\
Female, $\mathrm{n}(\%)$ & $54(94.7)$ & $48(92.3)$ & 0.707 \\
Body weight, $\mathrm{kg}$ & $51(44.5,56)$ & $49.8(45,55)$ & 0.656 \\
Cr-eGFR, mL/min/1.73m² & $67.9(59.3,77.2)$ & $68.55(60.2,78.7)$ & 0.853 \\
Value of serum calcium, mg/dL & $9.6(9.3,9.8)$ & $9.6(9.275,9.725)$ & 0.567 \\
Presence of vertebral fractures, $\mathrm{n}(\%)$ & $28(49.1)$ & $26(50)$ & 1.000 \\
Lumbar spine BMD, g/cm ${ }^{2}$ & $0.793(0.703,0.879)$ & $0.838(0.756,0.905)$ & 0.100 \\
Lumbar spine T score & $-2.6(-3.2,-2)$ & $-2.35(-2.9,-1.75)$ & 0.106 \\
Total hip BMD, g/cm ${ }^{2}$ & $0.665(0.585,0.701)$ & $0.641(0.587,0.679)$ & 0.439 \\
Total hip T score, mean (SD) & $-2.3(-2.9,-2)$ & $-2.5(-2.9,-2.175)$ & 0.424 \\
Femoral neck, g/cm ${ }^{2}$ & $0.615(0.56,0.67)$ & $0.617(0.576,0.682)$ & 0.430 \\
Femoral neck T score & $-2.8(-3.3,-2.3)$ & $-2.8(-3.2,-2.275)$ & 0.494 \\
P1NP, ng/mL & $67.2(51.6,81.4)$ & $68.9(46.2,84.4)$ & 0.911 \\
TRACP-5b, mU/dL & $581(445,684)$ & $511.5(420.5,656.5)$ & 0.105 \\
\hline
\end{tabular}

ZOL: zoledronic acid, APAP: acetaminophen, ELD: eldecalcitol, Q1: 25th percentile, Q3: 75th percentile, Cr-eGFR: estimated glomerular filtration rate calculated by creatinine, BMD: bone mineral density, P1NP: N-terminal propeptide of type I procollagen, TRACP-5b: tartrateresistant acid phosphatase- $5 b$ 
Table 2. Comparison between Patients with and without Adverse Events Regarding Demographic Characteristics at Baseline Using Univariate Analysis.

\begin{tabular}{lccc}
\hline \multicolumn{1}{c}{ Variables, median $(\mathrm{Q} 1, \mathrm{Q} 3)$} & With AE $(\mathrm{n}=39)$ & Without AE $(\mathrm{n}=70)$ & $\mathrm{p}$ value \\
\hline Age, years & $73(70,77.5)$ & $76.5(72,82)$ & 0.022 \\
Female, $\mathrm{n}(\%)$ & $38(97.4)$ & $64(91.4)$ & 0.418 \\
Body weight, $\mathrm{kg}$ & $50(46.5,56)$ & $50(44.5,55)$ & 0.742 \\
Cr-eGFR, $\mathrm{mL} / \mathrm{min} / 1.73 \mathrm{~m}^{2}$ & $67.9(55.95,76.05)$ & $68.8(60.3,78.15)$ & 0.450 \\
Value of serum calcium, mg/dL & $9.5(9.2,9.7)$ & $9.6(9.3,9.8)$ & 0.307 \\
APAP use, $\mathrm{n}(\%)$ & $8(20.5)$ & $31(44.3)$ & 0.021 \\
Presence of vertebral fractures, $\mathrm{n}(\%)$ & $19(48.7)$ & $35(50)$ & 1.000 \\
Lumbar spine T score & $-2.5(-3.2,-1.9)$ & $-2.55(-3,-1.8)$ & 0.786 \\
Total hip T score, mean (SD) & $-2.3(-2.9,-2.15)$ & $-2.4(-2.9,-1.925)$ & 0.665 \\
Femoral neck T score & $-2.8(-3.2,-2.35)$ & $-2.8(-3.2,-2.2)$ & 0.869 \\
\hline
\end{tabular}

Q1: 25th percentile, Q3: 75th percentile, AE: adverse events, Cr-eGFR: estimated glomerular filtration rate calculated by creatinine, APAP: acetaminophen

Table 3. Multivariate Analysis of Factors Associated with Adverse Events in Patients Treated with Zoledronic Acid.

\begin{tabular}{lccc}
\hline Variables & Crude OR $(95 \%$ CI $)$ & Adjusted OR (95\% CI) & p value \\
\hline Age & $0.93(0.88-0.99)$ & $0.93(0.88-0.99)$ & 0.017 \\
APAP use & $0.33(0.13-0.81)$ & $0.33(0.13-0.82)$ & 0.018 \\
\hline
\end{tabular}

OR: odds ratio, CI: confidence interval, APAP: acetaminophen

a

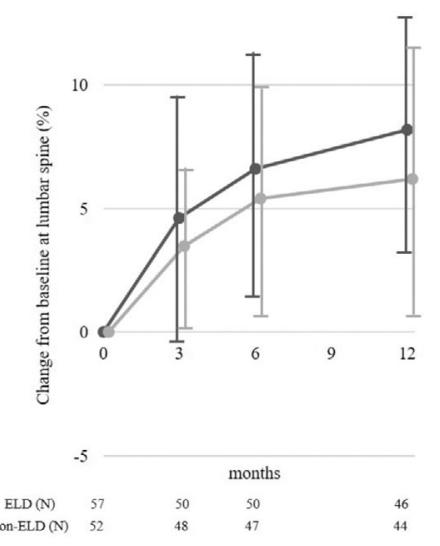

b

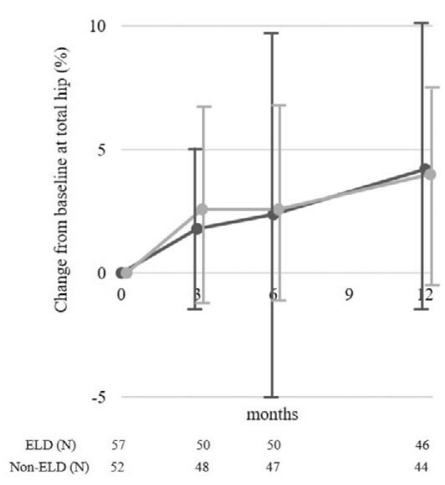

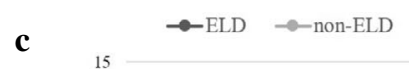

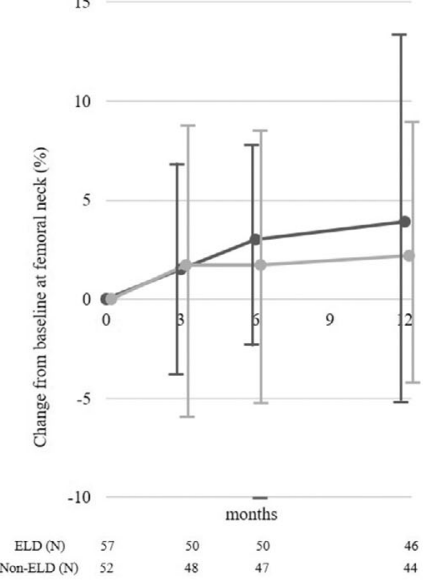

Figure 2. Percent changes from baseline in the bone mineral densities at the (a) lumbar spine, (b) total hip, and (c) femoral neck in the eldecalcitol and non-eldecalcitol groups at 3, 6, and 12 months.

nificantly decreased in all time points. However, $\triangle \mathrm{P} 1 \mathrm{NP}$ at 12 months $(\mathrm{p}=0.016)$ and $\triangle$ TRACP-5b at $3(\mathrm{p}=0.008), 6(\mathrm{p}<$ $0.001)$, and 12 months $(\mathrm{p}<0.001)$ significantly differed between the two groups.

\section{New vertebral fractures}

The rates of new vertebral fractures at 12 months were $15.2 \%$ in the ELD group and $6.5 \%$ in the non-ELD group. These rates did not differ significantly between the two groups $(\mathrm{p}=0.117)$.

\section{Discussion}

The present study revealed that the administration of APAP before ZOL infusion thrice daily for 3 days significantly reduced the rate of symptomatic adverse events in Japanese patients with primary osteoporosis. The common symptomatic adverse events after ZOL administration were pyrexia, myalgia, headache, arthralgia, malaise, and fatigue $(10,13,14)$. ZOL treatment for 2 years in Japanese patients with primary osteoporosis triggered the occurrence of pyrexia (39.3\%), arthralgia (16.2\%), myalgia (10.8\%), malaise $(9.0 \%)$, and headache $(7.5 \%)(13)$. In a previous 
study, the rate of adverse events observed in the ZOL group $(55.1 \%)$ was significantly higher than that in the intravenous ibandronate group (37.9\%) (18). Based on our results, ZOL combined with APAP was thus observed to reduce the number of symptomatic adverse events. One of the mechanisms of acute phase response associated with bisphosphonates was an evaluation of inflammatory cytokines (19). The levels of inflammatory cytokines such as tumor necrosis factoralpha and interleukin- 6 significantly increased at day 1 after ZOL infusion $(16,20)$. The administration of $650 \mathrm{mg}$ of APAP before ZOL infusion, four times daily for 3 days reduced the inflammatory cytokines levels at day 1 (16). In this study, age was identified as a factor associated with adverse events. We believe that ZOL combined with APAP treatment was effective for reducing the symptomatic adverse events in Japanese patients with primary osteoporosis.

In the present study, ZOL treatment significantly increased the BMD for the lumbar spine and total hip at 12 months. The efficacy rates did not affect ELD. In previous studies, $\triangle \mathrm{BMD}$ values for lumbar spine, total hip, and femoral neck at 12 months were $3.0-12.7 \%, 2.2-7.3 \%$, and 0.8 $7.7 \%$, respectively $(21-24)$. In the present study, the $\triangle \mathrm{BMD}$ values for lumbar spine, total hip, and femoral neck at 12 months in the ELD and non-ELD groups were $8.2 \%$ and $6.2 \%, 4.2 \%$ and $4.0 \%$, and $3.9 \%$ and $2.2 \%$, respectively. In osteoporotic patients receiving ELD treatment, the $\triangle \mathrm{BMD}$ values for lumbar spine, total hip, and femoral neck at 12 months were $2.51 \%, 1.50 \%$, and $1.95 \%$, respectively (25). In patients with primary osteoporosis receiving alendronate (ALN) plus ELD treatment, the $\triangle B M D$ values for lumbar spine, total hip, and femoral neck at 12 months were $7.3 \%$, $2.4 \%$, and $2.7 \%$, respectively. Comparing the ALN plus ELD group with the ALN plus vitamin D 400 IU plus Ca $610 \mathrm{mg}$ daily group, the $\triangle \mathrm{BMD}$ for femoral neck in former group was significantly larger than that in the latter group at 48 weeks (26). With regard to the efficacy of increasing the $\mathrm{BMD}$ of active vitamin $\mathrm{D}$, similar results were reported for denosumab treatment (27). Based on our results, no efficacy was observed regarding increased the BMD when ZOL was combined with ELD. The addition of ELD to ZOL treatment may not be necessary in the short term. In a previous report, an increased BMD at lumbar spine with alendronate treatment was associated with the 25-hydroxyvitamin D levels at baseline (28). However, we could not evaluate the 25hydroxyvitamin D levels at baseline in the present study. The 25-hydroxyvitamin $\mathrm{D}$ levels at baseline may affect the efficacy of ELD. The $\triangle \mathrm{P} 1 \mathrm{NP}$ and $\triangle \mathrm{TRACP}-5 \mathrm{~b}$ levels at 12 months in the ELD group were significantly more suppressed than those in the non-ELD group. With regard to turnover makers, the effects of ZOL combined with ELD were confirmed.

This study is associated with some limitations. First, in this study, all patients in the APAP group received ELD. The adverse events in the APAP group may have been due to ELD. However, in a previous report of large-scale post marketing surveillance in Japanese patients, the adverse drug reactions did not include pyrexia, arthralgia, headache, chest pain, and hyperemia (29). We believe that the effect of ELD on the adverse events in the APAP group was small. Second, the sample size was small and the study period was short. Therefore, a prospective study would be necessary to determine the most effective dose of APAP and the effect of larger sample sizes and longer periods of treatment.

In conclusion, this study demonstrated that ZOL treatment combined with APAP was effective in reducing symptomatic adverse events; ZOL increased BMDs of the lumbar spine and total hip. In the ELD and non-ELD groups, no difference was observed in the increase in the BMD of the lumbar spine, total hip, and femoral neck at 12 months. Based on our results, the administration of APAP should be considered as a therapeutic option to reduce symptomatic adverse events stemming from ZOL treatment in Japanese patients with primary osteoporosis, particularly in younger patients.

This study was approved by the independent ethics committee of Kamagaya General Hospital and was carried out following the principles of the Declaration of Helsinki.

Informed consent was obtained from the patients after explaining the study protocol.

\section{Author's disclosure of potential Conflicts of Interest (COI).} Takeshi Mochizuki: Honoraria, AbbVie, Astellas, Bristol-Myers, Chugai, Daiichi Sankyo, Eisai, Eli Lilly, Janssen, Mochida, Pfizer, Takeda and Tanabe-Mitsubishi. Koichiro Yano: Honoraria, AbbVie, Astellas, Ayumi, Bristol-Meyers, Eisai, Hisamitsu, Mochida and Takeda. Katsunori Ikari: Honoraria, AbbVie, Astellas, Bristol-Myers, Chugai, Eisai, Eli Lilly, Janssen, Takeda, TanabeMitsubishi and UCB.

\section{References}

1. Cooper C, Atkinson EJ, Jacobsen SJ, O'Fallon WM, Melton LJ 3rd. Population-based study of survival after osteoporotic fractures. Am J Epidemiol 137: 1001-1005, 1993.

2. Cauley JA, Thompson DE, Ensrud KC, Scott JC, Black D. Risk of mortality following clinical fractures. Osteoporos Int 11: 556-561, 2000.

3. Nishimura A, Akeda K, Kato K, et al. Osteoporosis, vertebral fractures and mortality in a Japanese rural community. Mod Rheumatol 24: 840-843, 2014.

4. Melton LJ 3rd, Therneau TM, Larson DR. Long-term trends in hip fracture prevalence: the influence of hip fracture incidence and survival. Osteoporos Int 8: 68-74, 1998.

5. Forsén L, Sogaard AJ, Meyer HE, Edna T, Kopjar B. Survival after hip fracture: short- and long-term excess mortality according to age and gender. Osteoporos Int 10: 73-78, 1999.

6. Muraki S, Yamamoto S, Ishibashi H, Nakamura K. Factors associated with mortality following hip fracture in Japan. J Bone Miner Metab 24: 100-104, 2006.

7. Hagino H, Endo N, Harada A, et al. Survey of hip fractures in Japan: recent trends in prevalence and treatment. J Orthop Sci 22: 909-914, 2017.

8. Siris ES, Harris ST, Rosen CJ, et al. Adherence to bisphosphonate therapy and fracture rates in osteoporotic women: relationship to vertebral and nonvertebral fractures from 2 US claims databases. 
Mayo Clin Proc 81: 1013-1022, 2006.

9. Rabenda V, Mertens R, Fabri V, et al. Adherence to bisphosphonates therapy and hip fracture risk in osteoporotic women. Osteoporos Int 19: 811-818, 2008.

10. Black DM, Delmas PD, Eastell R, et al. Once-yearly zoledronic acid for treatment of postmenopausal osteoporosis. N Engl J Med 356: 1809-1822, 2007.

11. Jacques RM, Boonen S, Cosman F, et al. Relationship of changes in total hip bone mineral density to vertebral and nonvertebral fracture risk in women with postmenopausal osteoporosis treated with once-yearly zoledronic acid $5 \mathrm{mg}$ : the HORIZON-Pivotal Fracture Trial (PFT). J Bone Miner Res 27: 1627-1634, 2012.

12. Boonen S, Reginster JY, Kaufman JM, et al. Fracture risk and zoledronic acid therapy in men with osteoporosis. N Engl J Med 367: 1714-1723, 2012.

13. Nakamura T, Fukunaga M, Nakano T, et al. Efficacy and safety of once-yearly zoledronic acid in Japanese patients with primary osteoporosis: two-year results from a randomized placebo-controlled double-blind study (zoledronate treatment in efficacy to osteoporosis; ZONE study). Osteoporos Int 28: 389-398, 2017.

14. Shiraki M, Tanaka S, Suzuki H, Ueda S, Nakamura T. Safety, pharmacokinetics, and changes in bone metabolism associated with zoledronic acid treatment in Japanese patients with primary osteoporosis. J Bone Miner Metab 35: 675-684, 2017.

15. Wark JD, Bensen W, Recknor C, et al. Treatment with acetaminophen/paracetamol or ibuprofen alleviates post-dose symptoms related to intravenous infusion with zoledronic acid $5 \mathrm{mg}$. Osteoporos Int 23: 503-512, 2012.

16. Silverman SL, Kriegman A, Goncalves J, Kianifard F, Carlson T, Leary E. Effect of acetaminophen and fluvastatin on post-dose symptoms following infusion of zoledronic acid. Osteoporos Int 22: 2337-2345, 2011.

17. Ebina K, Noguchi T, Hirao M, Kaneshiro S, Tsukamoto $Y$ Yoshikawa H. Comparison of the effects of 12 months of monthly minodronate monotherapy and monthly minodronate combination therapy with vitamin $\mathrm{K} 2$ or eldecalcitol in patients with primary osteoporosis. J Bone Miner Metab 35: 91-98, 2016.

18. Sieber $P$, Lardelli $P$, Kraenzlin CA, Kraenzlin ME, Meier C. Intravenous bisphosphonates for postmenopausal osteoporosis: safety profiles of zoledronic acid and ibandronate in clinical practice. Clin Drug Investig 33: 117-122, 2013.

19. Thiébaud D, Sauty A, Burckhardt $\mathrm{P}$, et al. An in vitro and in vivo study of cytokines in the acute-phase response associated with bisphosphonates. Calcif Tissue Int 61: 386-392, 1997.
20. Dicuonzo G, Vincenzi B, Santini D, et al. Fever after zoledronic acid administration is due to increase in TNF-alpha and IL-6. J Interferon Cytokine Res 23: 649-654, 2003.

21. Lyles KW, Colón-Emeric CS, Magaziner JS, et al. Zoledronic acid and clinical fractures and mortality after hip fracture. N Engl J Med 357: 1799-1809, 2007.

22. Cosman F, Eriksen EF, Recknor C, et al. Effects of intravenous zoledronic acid plus subcutaneous teriparatide [rhPTH(1-34)] in postmenopausal osteoporosis. J Bone Miner Res 26: 503-511, 2011.

23. Greenspan SL, Perera S, Ferchak MA, Nace DA, Resnick NM. Efficacy and safety of single-dose zoledronic acid for osteoporosis in frail elderly women: a randomized clinical trial. JAMA Intern Med 175: 913-921, 2015.

24. Tan W, Sun J, Zhou L, Li Y, Wu X. Randomized trial comparing efficacies of zoledronate and alendronate for improving bone mineral density and inhibiting bone remodelling in women with postmenopausal osteoporosis. J Clin Pharm Ther 41: 519-523, 2016.

25. Jiang $Y$, Tang $\mathrm{H}$, Ma X, et al. Eldecalcitol increases bone mineral density in Chinese osteoporotic patients without vitamin D or calcium supplementation. J Bone Miner Metab 37: 1036-1047, 2019.

26. Sakai A, Ito M, Tomomitsu T, et al. Efficacy of combined treatment with alendronate (ALN) and eldecalcitol, a new active vitamin D analog, compared to that of concomitant ALN, vitamin D plus calcium treatment in Japanese patients with primary osteoporosis. Osteoporos Int 26: 1193-1202, 2015.

27. Ebina K, Kashii M, Hirao M, et al. Comparison of the effects of denosumab between a native vitamin $\mathrm{D}$ combination and an active vitamin D combination in patients with postmenopausal osteoporosis. J Bone Miner Metab 35: 571-580, 2017.

28. Ishijima M, Sakamoto $Y$, Yamanaka M, et al. Minimum required vitamin D level for optimal increase in bone mineral density with alendronate treatment in osteoporotic women. Calcif Tissue Int $\mathbf{8 5}$ : 398-404, 2009.

29. Saito H, Kakihata H, Nishida $Y$, et al. The safety and effectiveness profile of eldecalcitol in a prospective, post-marketing observational study in Japanese patients with osteoporosis: interim report. J Bone Miner Metab 35: 456-463, 2017.

The Internal Medicine is an Open Access journal distributed under the Creative Commons Attribution-NonCommercial-NoDerivatives 4.0 International License. To view the details of this license, please visit (https://creativecommons.org/licenses/ by-nc-nd/4.0/).

(C) 2021 The Japanese Society of Internal Medicine Intern Med 60: 2585-2591, 2021 